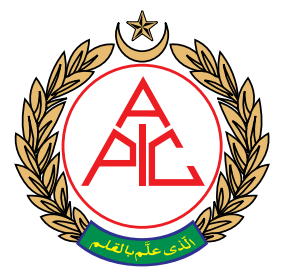

Coimbra Hospital and University Centre, Coimbra, (Portugal) Correspondence: Dr Natacha Andreia da Cunha Husson,

Rua Maria Vitória Bourbon Bobone n41 A501 3030-502 Coimbra, (Portugal)

Phone: 00351917864058

E-mail: natachahusson@gmail.com

Received: 15 October 2019,

Reviewed: 18 October 2019

Accepted: 19 October 2019

\title{
One shock after another; simulation can prevent fixation errors: A case report
}

\author{
Natacha Husson, MD, Claudia Carreira, MD, Nuno Babo, MD
}

\begin{abstract}
Introduction: Safety is essential in all the anesthesiologists' activity. Anesthesiology pioneered the use of simulation in training. Human factors play a big part in critical incidents. Understanding and identifying key cognitive errors specific to anesthesiology is the first step in metacognition training and strategies to prevent these errors and improve patient safety.
\end{abstract}

\begin{abstract}
A 64y female patient, ASA III, submitted to emergency laparotomy with right hemicolectomy. Surgery went uneventful until wound closure. After metamizole administration, the patient had had generalized skin rash, hypoxemia, bradycardia and hypotension, treated as an anaphylactic shock. In the recovery room, she had another episode of severe She had cardiac arrest. Advanced life support was successful. Ecofast (acronym for "The Focused Abdominal Sonography for Trauma Scan") revealed intraperitoneal fluid compatible with massive hemoperitoneum. Hemorrhagic shock was treated with exploratory laparotomy and hemodynamic support with progressive improvement.

It is important to note that a possible fixation error could have delayed the diagnosis of the hemorrhagic shock that overlapped the first anaphylactic shock and culminated in cardio-respiratory arrest. Fixation errors occur when one focuses only on one factor rather than other equally relevant and more predictable ones. Training through simulation increases awareness of potential problems in routine and non-routine settings and allows faster skill acquisition and recognition of problems. ACRM programs where the CRM model is applied to anesthesiology, allows the teaching and training of team behaviors in crisis situations.
\end{abstract}

Key words: Anesthesiology; Human factors; Fixation errors; Simulation; Emergency; Anaphylactic shock; Hemorrhagic shock

Citation: Husson N, Carreira C, Babo N. One shock after another; simulation can prevent fixation errors: A case report. Anaesth pain \& intensive care 2019;23(4):401403. DOI: 10.35975/apic.v23i4.1179

\section{INTRODUCTION}

Anesthesiologists need to perform a broad number of tasks in order to maintain a patient's physiological stability, and require a solid foundation in pharmacology, medicine and surgery, as well as the ability to handle complex situations and perform invasive procedures. All of the above require excellence in performance to ensure safety, and thus comparisons between anesthesiology and aviation have been made..$^{1,2}$ Both fields use simulation on a regular basis in their training. ${ }^{1,2}$ Anesthesiology pioneered the use of simulation-based training in the medical field. This began with very basic simulators and is performed today with high-fidelity simulators that accurately recreate human anatomy and physiology in settings validated accordingly. ${ }^{1,3}$

Medical error is currently emerging as a serious health issue and anesthesiology is not immune to this predicament. Human factors, mostly cognitive errors, involving subconscious biases and faulty-thought processes, play a part in critical incidents. ${ }^{4,5} \mathrm{~A}$ review of decision-making psychology and cognitive errors has not yet been published in the contemporary 
anesthesiology literature. ${ }^{4}$ Understanding and identifying key cognitive errors specific to anesthesiology is the first step in metacognition training and in creating strategies to prevent these errors and improve patient safety. ${ }^{4,5}$

It is widely accepted that human factors, whether individual or collectively as a team, influence behavior and can affect safety at work. ${ }^{2,6}$ Understanding the limitations of human performance such as tiredness, fatigue or stress in both cognition and social relationships is therefore essential for error reduction. ${ }^{2,5}$ Anesthesiologists' performance can be affected by physical and mental tiredness, stress, especially in out-of-routine events and work in complex environments. The coercion to reduce time and costs of one's activity may also play a role in this scenario. ${ }^{5,6}$ Fixation error is one sort of human error which can be overcome by repeated training in simulated scenarios. This case report highlights the importance of recognition of fixation error and the simulation training.

\section{CASE REPORT}

Our ethical committee informed us that for publishing a case report without any personal patient information and without any experimental procedures, we would only need the patient's informed consent, which was obtained. A case of a female patient of $64 \mathrm{y}$, ASA III, with history of type 2 diabetes, nephrolithiasis and colon cancer with liver metastasis presented with intestinal occlusion and was scheduled for emergency laparotomy with right hemicolectomy. Chronic medication included propranolol, omeprazole and metformin. No allergies were known.

Rapid sequence induction was achieved with etomidate $20 \mathrm{mg}$ and succinylcholine $50 \mathrm{mg}$. Maintenance was ensured through controlled ventilation with sevoflurane and fentanyl. Relaxation was maintained with cisatracurium $10 \mathrm{mg}$.

Surgery was uneventful until wound closure, when inj. metamizole $2 \mathrm{~g}$ was administered in $20 \mathrm{ml}$ saline. Five minutes after, the patient developed generalized skin rash, hypoxemia, bradycardia and hypotension (mean arterial pressure $\leq 40 \mathrm{mmHg}$ ). Adrenaline 0.5 mg IM was administered, $\mathrm{FiO}_{2}$ was increased to $100 \%$ and the perfusion rate of ongoing fluids increased, with no significant response. Hydrocortisone $200 \mathrm{mg}$, ranitidine $50 \mathrm{mg}$, clemastine $2 \mathrm{mg}$ and second bolus of adrenaline $0.5 \mathrm{mg}$ IM were administered, with hemodynamic parameters improvement. After radial artery and femoral vein cannulation, a sample was taken for serum tryptase measurement. The patient was stabilized and transferred to the recovery unit, intubated and sedated with midazolam and morphine due to airway edema.
In the recovery unit, she once more developed severe hypotension and tachycardia, whilst maintaining edema and a generalized skin rash. Adrenaline perfusion was initiated with refractory hemodynamic response. At this stage the patient arrested. Advanced life support was successful at the first cycle. Due to an abdominal distention USG was performed revealing free intraperitoneal fluid, compatible with massive hemoperitoneum.

Exploratory laparotomy was performed and revealed active bleeding from the colic artery, which was controlled and hemostasis was revised. Patient was transfused with a total of eight units of erythrocyte concentrate, four units of fresh frozen plasma, and tranexamic acid $1 \mathrm{~g}$, with progressive hemodynamic improvement.

The patient entered the recovery room sedated, on controlled ventilation. She presented favorable recovery without amine perfusion and was extubated after six hours, with a GCS of 15.

\section{DISCUSSION}

The case report depicts a situation in which a possible fixation error (shock due to anaphylaxis to metamizole) would have delayed the diagnosis of the hemorrhagic shock that overlapped the first and culminated in cardio-respiratory arrest. In the context of emergency surgery and after an emergent event, e.g. anaphylactic shock, fixation error is quite predictable when a second emergent event occurs within a short time.

Fixation errors occur when the anesthesiologist focuses only on one data rather than other equally relevant data, due to associations made with past experiences and making it difficult to find new ways of resolving the present condition. ${ }^{7}$ Some errors might be more frequent among less experienced anesthesiologists, while others are more frequent among more experienced ones. ${ }^{4}$

Mistakes due to tiredness, inability to stay focused, reduced motivation, difficulty in solving problems, confusion, irritability, poor communication, poor information processing, and decreased ability to react are also frequently observed. ${ }^{5,6}$

As anesthesiologists are frequently exposed to numerous sensory inputs, they automatically respond to the relevant ones, allowing work to be efficient and ignore the unimportant inputs. ${ }^{8}$ However, relevant, inputs may be mistakenly blocked out and attention deficit becomes difficult to avoid when the event is unexpected, unnoticed or concurrent with other tasks, distractions, intellectual overload, stress or tiredness. ${ }^{8}$

Increasing awareness has been the proposed strategy 
for problem solving in anesthetic practice and its training allows for the development of the necessary skills to avoid fixation errors. ${ }^{5,6,7}$ Training through simulation increases awareness of potential problems in routine and non-routine settings and allows faster skill acquisition and recognition of problems. ${ }^{7}$

Inattentional blindness (IB) is a human factor that largely contributes to the lack of awareness and to the inattentiveness to important but unexpected events when performing a different task. ${ }^{8}$ IB can be affected by the conditioning of the fact that anesthesiologists to focus on the causes and treatment of expected changes. ${ }^{8}$ Anesthesiologists are aware of the dangers of overloading sensory data, information and tasks, as well as tiredness and distractions present in an operating room, which predispose to IB. ${ }^{8}$ Many errors are due not to a lack of knowledge or of technical skills, but to lack of perception of failure (IB), which may be related to fixation errors that occur when the clinician focuses only on certain portions of data. ${ }^{8}$

To deal with unusual situations, anesthesiologists must be able not only to recognize the clinical needs of those situations, but also to plan an approach to their resolution. ${ }^{7}$ In simulation scenarios, hands-on experience significantly contributes to reinforce skills in performing procedures. Also, scenario observer feedback and debriefing issues allow for reflective and solid learning. ${ }^{1}$

Anesthesia Crisis Resource Management (ACRM) programs where the crisis resource management (CRM) model is applied to anesthesiology, allow for the teaching and training of team behaviors in crisis situations, addressing interpersonal communication, decision making and team management, improving their performance in clinical practice. ${ }^{1,5,6}$ Effective communication is essential to the smooth functioning of the team in emergency situations. ${ }^{6}$

Non-technical skills (NTS) are a very significant and important component of the anesthesiologist's daily life, which should be included in their training, and can be divided into four categories; situation awareness, decision-making, task management, and leadership and teamwork. . $^{1,2,5,6,8}$ Also in this field of NTS, simulation offers a way of acquiring and training these skills in a controlled manner, being a great tool for professional development of anesthesiologists. ${ }^{1,2}$

Thus, the current literature reiterates that, human error is a reality in clinical practiceand that unexpected, emergent events under certain circumstances favor its occurrence. Anesthesiology pioneered in the use of simulation and repeated training to cope with emergency scenarios and exceptional situations, as it represents a medical specialty in which safety plays a very important role given the complexity of anesthesiologists' decisions and tasks. Simulation in the training of anesthesiologists can play a key role in their professional development. Therefore, investment in this area should be continued.

Conflict of interest: None declared by the authors

\section{Authors' contribution:}

$\mathrm{NH}$ : Concept, literature search, conduction of the study work and manuscript editing

CC: Concept, conduction of the study work and manuscript editing

NB: Manuscript editing

\section{REFERENCES}

1. Bhagwat M. Simulation and anaesthesia. Indian J Anaesth 2012;56 (1):14-20. [PubMed [Free full text] DOI: $10.4103 / 0019-5049.93338$

2. Galvin R, Flin R. Review article: the influence of psychology and human factors on education in anesthesiology. Can J Anesthe 2012;59:151-158. [PubMed] [Free full text] DOI: 10.1007/s12630-011-9634-z

3. Gaba DM, Howard SK, Fish KJ, Smith BE, Sowb YA. Simulation-based training in anesthesia crisis resource management (ACRM): a decade of experience. Simulation \& Gaming 2001;32 (2):175-193. [Free full text] DOI: $\underline{10.1177 / 104687810103200206}$
4. Stiegler MP, Neelankavil JP,Canales C, Dhillon A. Cognitive errors detected in anaesthesiology: a literature review and pilot study. Br J Anaesth 2012;108 (2):229-235. [PubMed] [Free full text] DOl: $10.1093 /$ bja/aer387

5. Chandran R, DeSousa KA. Human factors in anaesthetic crisis. World J Anesthesiol 2014;3 (3):203-212. [Free full text] DOl: 10.5313/wia. v3.i3.203

6. Jones CPL, Fawker-Corbett J, Groom P, Morton B, Lister C, Mercer SJ. Human factors in preventing complications in anesthesia: a systematic review. Anesthesia 2018;73 (suppl.1):12-24. [PubMed] [Free full text] DOI:

\subsection{1/anae.14136}

7. Fioratou $E$, Flin $R$, Glavin R. No simple fix for fixation errors: cognitive processes and their clinical applications. Anaesthesia 2010;65:61-69. [PubMed] [Free full text]

8. Ho AMH, Leung JYC, Mizubuti GB, Contardi LH, Chan MTV, Lo TSF, et al. Inattentional blindness in anesthesiology: a simulation study. Journal of Clinical Anesthesia 2017;42:36-39. [PubMed] [ [Free full text] DOI: 10.1111/i.13652044.2009.05994.x 